\title{
Quantitative analysis of beta-actin, beta-2-microglobulin and porphobilinogen deaminase $m R N A$ and their comparison as control transcripts for RT-PCR
}

\author{
J. Lupberger, K.-A. Kreuzer, G. Baskaynak, U. R. Peters, P. le Coutre and \\ C. A. Schmidt* \\ Department of Medicine, Division of Hematology/Oncology, Charité Virchow-Klinikum, \\ Humboldt University Berlin, Augustenburger Platz 1, Berlin, FRG
}

(Received 2 July 2001; accepted for publication 24 September 2001)

\begin{abstract}
Quantitation of target mRNAs using the reverse-transcription polymerase chain reaction found a widespread field of application in diverse biomedical diagnostic assays. However, the problem of varying sample quality has to be solved by correcting target molecule amounts through detection of an endogenous control template. The choice of an appropriate reference gene is still object of debate as pseudogene co-amplification and expression level variations may limit the usefulness of some currently used reference reactions. We compared quantitative expression levels of the commonly used endogenous reference genes beta-actin ( $\beta$-actin), beta-2-microglobulin $\left(\beta_{2}-M G\right)$ and porphobilinogen deaminase (PBDG) using the TaqMan chemistry. With these assays we investigated the respective expression patterns in K562 cells and leucocytes of normal individuals as well as of malignoma patients. In K562 cells $1544+246 \beta$-actin, $65 \pm 30 \quad \beta_{2}$-MG and $22 \pm 8$ PBDG copies/cell were detected. In normal leucocytes $491 \pm 97 \beta$-actin, $40 \pm 17 \beta_{2}$-MG and $<1$ PBDG copies/cell were quantified. Leucocytes of various malignancies exhibited $84 \pm 51 \beta$-actin, $106 \pm 8 \beta_{2}-\mathrm{MG}$ and $<1$ PBDG copies/cell. We conclude that $\beta_{2}-\mathrm{MG}$ is the most suitable reference gene tested as its variation between different sample origins and within distinct cell types was acceptable low.
\end{abstract}

(c) 2002 Elsevier Science Ltd.

KEYWORDS: reverse transcription polymerase chain reaction, controls, reference genes, betaactin, beta-2-microglobulin, porphobilinogen-deaminase.

\section{INTRODUCTION}

Real-time fluorescence PCR provides a highly sensitive, reproducible and fast way for the quantitation of nucleic acid sequences. Since it was first described in 1995 an increasing number of assays using this technique are going to replace previous quantitative PCR methods in routine laboratories.

Its basic principle is the usage of a non-extendable fluorescent probe, which is complementary to a specific sequence within the target sequence. On its terminal nucleotides it is labelled with different fluorescent dyes. The quencher dye, which is typically covalently attached to the 3 '-terminus suppresses fluorescent emission of the reporter dye, located at the 5'-terminus. This phenomenon is called the 'Fluorescence Resonance Energy Transfer' $(\text { FRET })^{1,2}$ and is based on the close proximity of both dyes resulting in a low measurable fluorescent signal.

\footnotetext{
* Author to whom all correspondence should be addressed: Priv.-Doz. Dr. med. C.A. Schmidt, Department of Medicine, Division of Haematology/Oncology, Charité Virchow-Klinikum, Humboldt University Berlin, Augustenburger Platz 1, 13353 Berlin, FRG, Tel. +49 (030) 450-553663; Fax. +49 (030) 450-559929; E-mail: christian.schmidt@ charite.de
} 
In case of hybridization to the target sequence the Taq DNA polymerase cleaves the probe from the strand during extension of the primers via its $5^{\prime}-3^{\prime}$ exonuclease activity. ${ }^{3}$ Thus, the distance between both dyes increases and the reporter dye starts to emit a detectable fluorescent signal whose accumulation is proportional to the starting amount of cDNA in the reaction. Compared to former semi-quantitative approaches the advantage of such real time PCR techniques is the detection at the log-phase of the amplification where no limiting reaction factors might influence the reaction kinetics.

In general, a major problem of cDNA synthesis is the sensitivity and potential degradation of RNA during the entire sample processing. Thus, varying sample qualities need to be corrected by co-amplification of an internal standard. The commonly used endogenous references belong to the group of 'housekeeping genes'. In an ideal setting these genes are constitutionally expressed by all cell types and should not be affected by any human disease. But even widely used reference genes like beta-actin, glyceraldehyde-3-phosphate or hypoxanthine phosphoribosyltransferase (HPRT) ${ }^{4}$ were criticised in the past because of possible pseudogene co-amplifications. Furthermore, actual transcription rates of most reference RNA transcripts with respect to different sampling procedures and various diseases are still unknown.

In order to evaluate which reference genes may be suitable for control reactions using the so called TaqMan chemistry we developed absolute quantitative RT-PCR assays for beta-actin ( $\beta$-actin), beta-2microglobulin $\left(\beta_{2}-M G\right)$ and porphobilinogen deaminase (PBGD). Furthermore, in order to study their expression patterns in vitro and in vivo we determined their amount in K562 cells, normal peripheral blood cells and peripheral leucocytes of malignoma patients.

\section{MATERIALS AND METHODS}

The following samples were investigated: separate RNA extractions of K562 cells $(n=4)$, normal leucocyte preparations $(n=4)$ as well as peripheral blood leucocytes of chronic myelogenous leukaemia $(n=$ $1)$, chronic lymphocytic leukaemia $(n=1)$, acute myelogenous leukaemia $(n=1)$, mamma carcinoma $(n=$ $1)$ B-cell non-Hodgkin lymphoma $(n=2)$, multiple myeloma $(n=2)$ and colon carcinoma $(n=2)$. The K562 cell line grew in 90\% RPMI 1640 medium including glutamax-I and 10\% fetal bovine serum (Life Technologies, Karlsruhe, Germany). To minimize cell cycle variations samples were all taken during the log phase of growth. All extractions were performed immediately after collection with a maximum processing time of three hours until storage of extracted RNA $\left(-80^{\circ} \mathrm{C}\right)$. Peripheral blood samples were collected into sterile heparin tubes (Sarsted, Numbrecht, Germany). After centrifugation for $5^{\prime}$ at $400 \times \mathbf{g}$ all leukocytes from the blood pellet were separated by selective erythrocyte lysis (QIAmp RNA Blood Mini Kit; Qiagen, Hilden, Germany). Pellets were resuspended in phosphate buffered saline (PAA Laboratories, Linz, Austria) and filtrated through a $40 \mu \mathrm{m}$ cell strainer (Becton Dickinson, Franklin Lakes, USA) in order to remove larger cell precipitations after the resuspension of the pellet. Cell density was subsequently assessed using an automated cell counter (Casy 1; Schärfe Systems, Reutlingen, Germany) and the sample were diluted to a cell concentration of $2-4 \times 10^{5}$ per $\mathrm{ml}$. With regard to potential deviations in terms of different cell densities we performed RNA extractions of one cell sample at three different cell dilutions in parallel. Thus, 9 different samples were obtained from one origin: $3 \times 10^{6}, 3 \times 10^{5}$ and $3 \times 10^{4}$ cells (QIAmp RNA Blood Mini Kit; Qiagen, Hilden, Germany) and suspended in $30 \mu \mathrm{l}$ Tris/ $\mathrm{HCl}$ buffer. A maximum amount of $1000 \mathrm{ng}$ cell line RNA and $8 \mu \mathrm{l}$ of blood RNA were reverse transcribed (RT) into cDNA. The RT mixture contained $100 \mu \mathrm{M}$ random hexamer primers $\left(\mathrm{pd}(\mathrm{N})_{6}\right.$; Amersham Pharmacia Biotech, San Francisco, USA), $2 \cdot 5 \mathrm{mM}$ dNTP (Life Technologies, Karlsruhe, Germany), $10 \times$ PCR buffer (Life Technologies, Karlsruhe, Germany), $0.1 \mathrm{M}$ dithiotreitol (DTT; Life Technologies, Karlsruhe, Germany). After an initial denaturation ribonuclease inhibitor (recombinant RNasin; Promega, Madison, USA) and RNA polymerase (SuperScript RT; Life Technologies, Karlsruhe, Germany) was added. cDNA was stored at $-20^{\circ} \mathrm{C}$ until being assayed.

We performed TaqMan PCR with a single pair of primers for every reference gene separately. For $\beta$ actin primer oligonucleotides were designed to hybridize within the $5^{\prime}$-untranslated region of exon 1 (sense) and within exon 2 (antisense) of human $\beta$ actin cDNA. ${ }^{5}$ The sense primer of $\beta_{2}-\mathrm{MG}$ was within exon 1 and 2 (5'-TACATgTCTCgATCCCACTTAACTAT) and the antisense primer within exon 2 and $3\left(5^{\prime}-\right.$ AgCgTACTCCAAAgATTCAggTT) of human $\beta_{2}$-MG cDNA. The intron spanned primer sequences prevented genomic DNA co-amplification.

For $\beta_{2}$ MG we used the following fluorescent probe oligonucleotide: 5'-CTCACgTCATCCAgCAgAgAATggAAAgTCA, which was labelled at the $5^{\prime}$-terminus with the reporter dye 6-carboxy-fluorescein phosphoramidite (FAM) and 6-carboxy-tetramethyl-rhodamine (TAMRA) at the 3 '-terminus as the quenching dye. The sense primer for PBGD was located within 
exon 1 and 2 (5'-TgCAACggCggAAgAAAACA), and the antisense primer in exon 7 (5'-AgATggCTCCgATgg) of human PGBD cDNA. Here the probe sequence was 5'-CCAAAgATgAgAgTgATTCgCgTg, also labelled with the reporter dye FAM ( $5^{\prime}$ - terminus) and the quenching dye TAMRA ( $3^{\prime}$-terminus). All oligonucleotides were synthesised by TIB Molbiol Inc (Berlin, Germany).

For TaqMan PCR calibration we generated a calibrator dilution series for each reference gene ranging from $10^{7}$ to 10 copies per $2 \mu$ l. The required amount of specific cDNA was amplified from RT material of healthy human blood with specific primers. In case of $\beta_{2}$-MG we performed plasmid specific PCR after cloning the target sequence into the pCR2.1-TOPO vector (TOPO TA Cloning Kit; Invitrogen Corp., Carlsbad, USA) using the M13 priming site on the LacZ $\alpha$ fragment. After extraction from a 3\% agarose gel (QIAquick Gel Extraction Kit; Qiagen, Hilden, Germany) the optical density was measured at $260 \mathrm{nM}$ in a photometer and the number of copies was calculated. On every TaqMan PCR plate all the calibration standards and CDNA samples were detected in duplicates using the ABI PRISM 7700 Sequence Detection System (Perkin-Elmer Applied Biosystems, Foster City, USA) over 45 PCR cycles.

\section{RESULTS}

During TaqMan PCR fluorescence kinetic of a given sample follows a sigmoid growth function (Fig. 1A). The cycle when the signal exceeds a set threshold value (Ct; threshold cycle) is directly correlated to the calculated copy value of these dilution. Thus, the absolute copy amount of an unknown DNA sample can be calculated from the regression curve of the logarithmic scaled standard $\mathrm{Ct}$ values against the linear scaled standard copy amounts (Fig. 1B). With our approach we obtained correlation coefficients $(r)$ for beta-actin of 0.999 , for $\beta_{2}-\mathrm{MG}$ of 0.999 and for PBGD of 0.998 . Figure 1C shows the corresponding results of the used standard series on a 3\% agarose gel. All assays were able to amplify a minimum amount of 10 copies target cDNA per $2 \mu \mathrm{l}$ sample. Intra-assay coefficients of variation (CV) were $6 \%(\beta$ actin), $10 \%\left(\beta_{2}-\mathrm{MG}\right)$ and $27 \%$ (PBGD). Inter-assay CV were $7 \%$ ( $\beta$-actin), 3\% ( $\beta_{2}$-MG) and $16 \%$ (PBGD), as calculated by variation from the mean of three separate measurements of the same cDNA. Genomic DNA samples remained steadily negative over 45 cycles of TaqMan PCR. Absolute copy numbers were calculated back to the initial cell numbers, which were set into the RNA extraction. As a result we obtained a copy/cell-ratio representing the average target RNA amount per cell. In Figure 2 this ratio is shown obtained from the different cell types. The average transcript level of beta-actin in the $\mathrm{K} 562$ extractions was 1544 copies/cell (sD: 246 copies/cell), 491 copies/cell (sD: 97 copies/cell) in the healthy blood donors and 84 copies/cell (sD: 51 copies/cell) in leucocytes derived from different cancer patients was. Differences between the cell types were significant as assessed by Students' $t$-test $(P<0 \cdot 001)$. The average transcript amount of $\beta_{2}-\mathrm{MG}$ in $\mathrm{K} 562$ was 65 copies/cell (sD: 30 copies/cell), 40 copies/cell (sD: 17 copies/cell) in normal leucocytes and 106 copies/cell (sD: 8 copies/cell) in cancer patients ( $P$-values between each group was $>0.05$ but $<0.001$ between K562 cells and the cancer patients; n.s.). For PBGD, the average expression level of K562 was 22 copies/cell (sD: 8 copies/cell), 0.007 copies/cell (sD: $0 \cdot 003$ copies/ cell) in healthy blood donors leucocytes and $0 \cdot 001$ copies/cell (sD: 0.0009 copies/cell) in leucocytes of the cancer patients. The $P$-value between healthy blood donors and cancer patients was $>0.001$ (n.s.), while means were significantly different between K562 cells, normal leucocytes and cancer patient leucocytes $(P<0 \cdot 001)$.

\section{DISCUSSION AND CONCLUSIONS}

The advantages of the TaqMan method regarding absolute quantification of DNA sequences can also be used for cDNA samples if appropriate control reactions are performed. However, this can be complicated by altered reference gene transcription within one individual or between different sample material. ${ }^{6}$ In search of a suitable control target we investigated three widely used reference genes with respect to their expression stability. We found comparable transcription levels within and between all samples for $\beta_{2}$-MG and conclude that it is the most suitable endogenous reference from those tested. PBGD although shows a comparable average transcription rate in healthy blood donors and different cancer patients, but in the latter case with relatively high variations and with a very low absolute expression ( $<1$ copies/cell). The differences of $\beta$-actin in all tested cells are acceptable, but, surprisingly, the degree of its expression varies from K562 cells > cancer patients $>$ normal donors.

Furthermore, $\beta_{2}-\mathrm{MG}$ appears to be expressed in a suitable amount per cell. As one single PBGD RNA transcript does represent less than one cell it can be speculated that it does not fulfil the criteria of an 'housekeeping gene' which is expected to be expressed by every single cell. Possibly, PBGD transcription is restricted to certain subpopulations of 


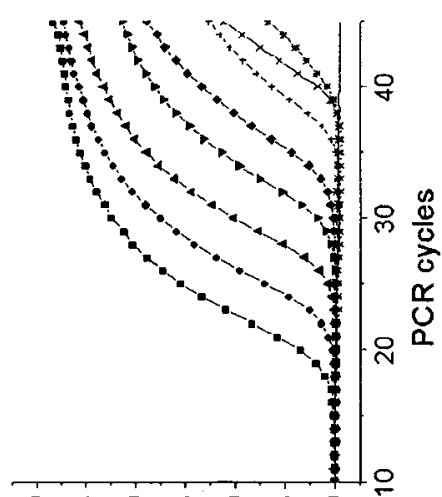

일 К!

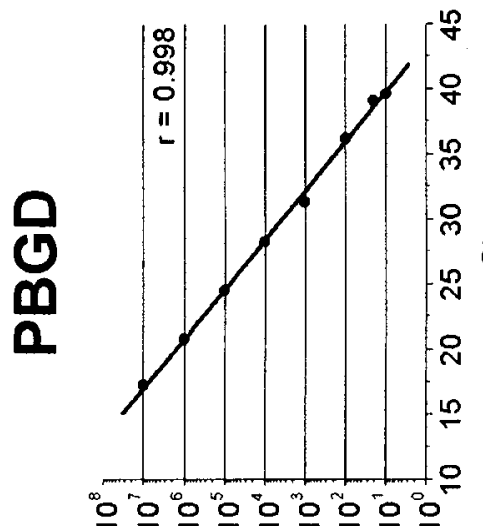

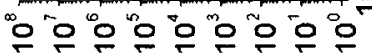

$7 \mathrm{rl} / \mathrm{sol}$ doo

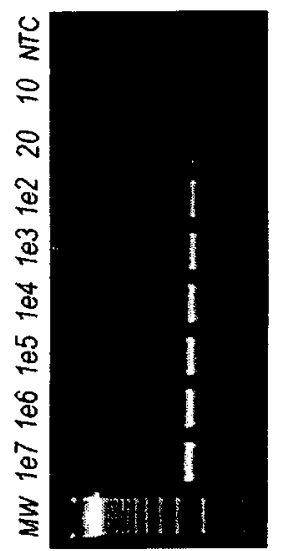

ㅎํ:

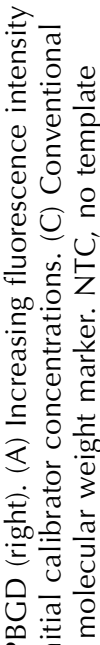

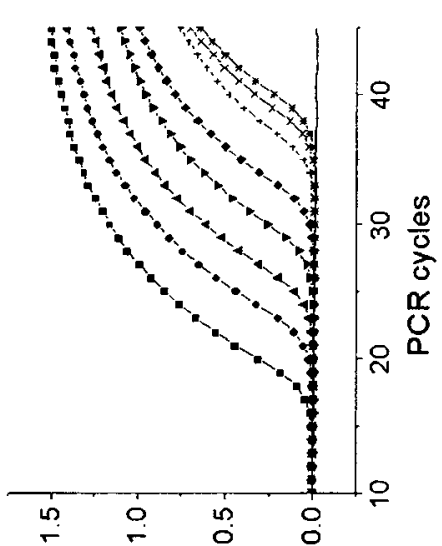

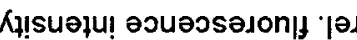

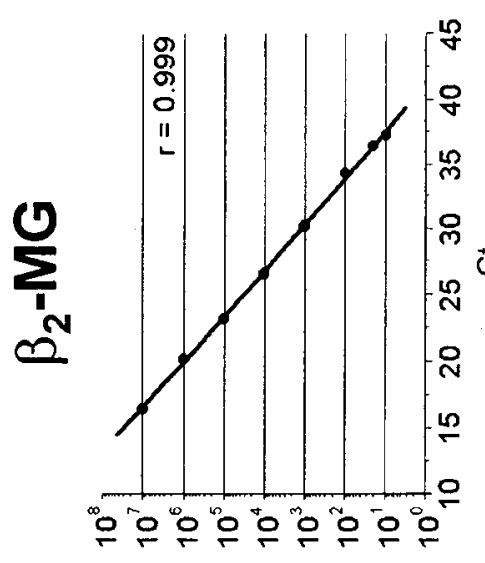

$7 \mathrm{r} / \mathrm{s}$ I!dos

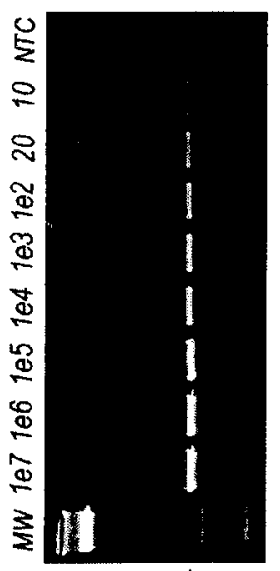

มื.s

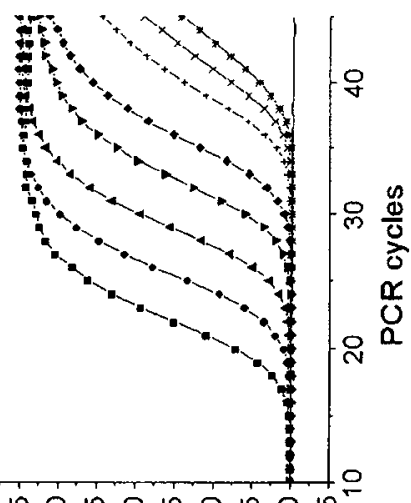

0 Ln 0 un 0 un 0 ln o un

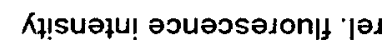

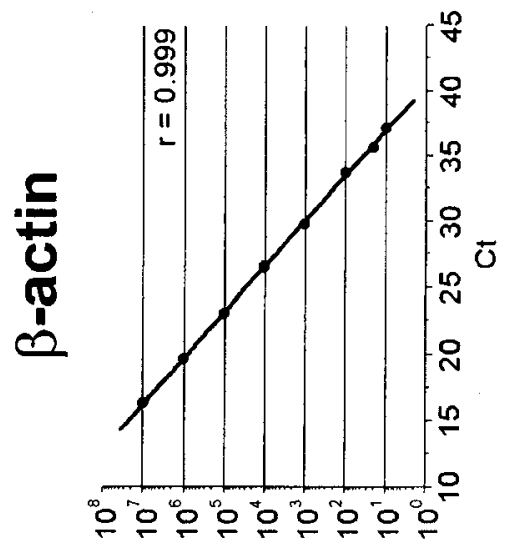

$m$

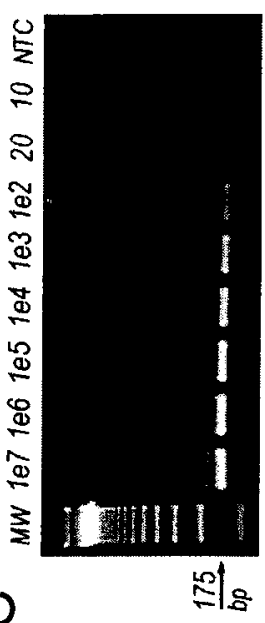

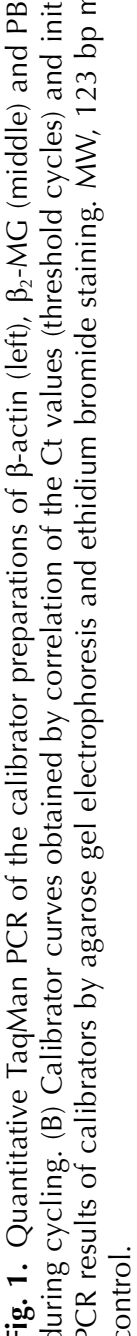




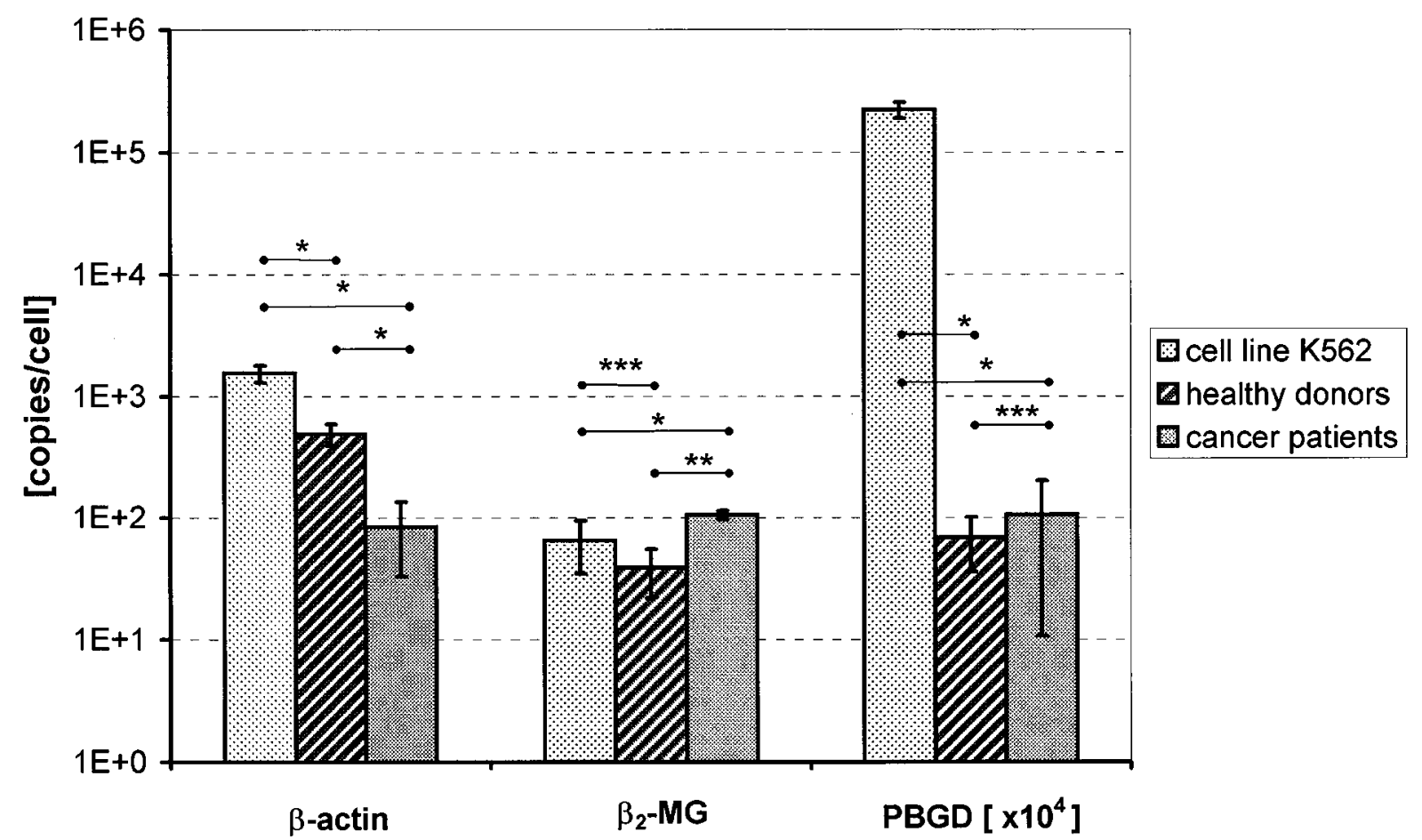

Fig. 2. Expression of reference genes in $\mathrm{K} 562$ cells, leucocytes of healthy blood donors and leucocytes of various cancer patients. For graphical reasons absolute amounts of PBGD transcripts are multiplicated by $10^{4}$. $P$-values of significance: $*<0 \cdot 001 ; * *<0 \cdot 01 ; * * *>0 \cdot 05$.

leucocytes. Finally, we were able to detect $\beta$-actin and $\beta_{2}-M G$ transcripts in as little as 10000 cells. Even if not representative for a sufficient number of cells these genes can be used to detect the blank existence of a minimum of $10^{4}$ cell/per reaction.

It was claimed that RT-PCR has a sensitivity of 1 molecule per $10^{6}$ cells. However, this is an theoretical assumption as the RNA content of $10^{6}$ cells $(5-8 \mu \mathrm{g})^{7}$ cannot be analyzed in a single reaction. Thus, an amount of cDNA must be defined which represents a minimum number of cells. According to our observation $10^{5}$ cells harbour $4.9 \times 10^{7} \beta$-actin copies or $3.9 \times 10^{6} \quad \beta_{2}$-MG copies (healthy blood donors). We therefore suggest that these molecule numbers should be considered to guarantee that the quantitative results represent at least $10^{5}$ cells.

It is now of interest whether all leucocyte types contribute equally to the absolute amount of reference genes which again is obligatory for constitutionally expressed control genes. Furthermore, their contribution within and between different tissues is of importance for future investigations. Further studies should aim to clarify this and possibly to identify superior control RNAs.

\section{ACKNOWLEDGEMENTS}

This work was supported by a grant from the Deutsche José Carreras Leukämie-Stiftung (DJCLS-R22).

\section{REFERENCES}

1. Livak, K. F., Flood, S. J., Marmaro, J., Giusti, W. \& Deetz, K. (1995). Oligonucleotides with fluorescent dyes at opposite ends provide a quenched probe system useful for detecting PCR product and nucleic acid hybridization. PCR Methods and Applications 6, 35762.

2. Förster, V. T. (1948). Zwischenmolekulare Energiewanderung und Fluoreszenz. Annals of Physics 2, 5575.

3. Holland, P. A., Abramson, R. D., Watson, R. \& Gelfand, D. H. (1991). Detection of specific polymerase chain reaction product by utilizing the $5^{\prime}-3^{\prime}$ exonuclease activity of Thermus aquaticus DNA polymerase. Proceedings of the National Academy of Science of the United States of America 88, 7276-80.

4. Sellner, L. T. \& Turbett, G. R. (1996). The presence of a pseudogene may affect the use of HPRT as an endogenous mRNA control in RT-PCR. Molecular and Cellular Probe 10, 481-3. 
5. Kreuzer, K. A., Lass, U., Landt, O., Nitsche, A., Laser J., Ellerbrok, H. et al. (1999). Highly sensitive and specific fluorescence reverse transcription-PCR assay for the pseudogene-free detection of beta-actin transcripts as quantitative reference. Clinical Chemistry 45, 297-300.

6. Wu, Y. Y. \& Rees, J. L. (2000). Variation in epidermal housekeeping gene expression in different pathological states. Acta Dermato-Venereologica 80, 2-3.

7. Ausubel, F. M., Brent, R., Kingston, R. E., Moore, D. D., Seidman, J. G., Smith, J. A. et al.Current Protocols in Molecular Biology, Suppl. 36. Harvard Medical School: John Wiley \& Sons: 4.2.9. 